D.O.I.: $10.3895 / \mathrm{S} 1808-04482009000400012$

\title{
APLICACIÓN DE ANÁLISIS DE FACTORES PARA IDENTIFICAR DIMENSIONES DE CALIDAD EN ESTUDIANTES DE INGENIERÍA INDUSTRIAL
}

\section{APPLICATION OF FACTORS ANALYSIS TECHNIQUE TO IDENTIFY QUALITY DIMENSIONS IN INDUSTRIAL ENGINEERING STUDENTS}

\author{
Carlos Torres ${ }^{1}$; Elizabeth Sepúlveda ${ }^{2}$ \\ ${ }^{1}$ Universidad del Bío-Bío - Departamento de Ingeniería Industrial - Concepción - Chile \\ ctorres@ubiobio.cl \\ ${ }^{2}$ Universidad del Bío-Bío - Departamento de Ingeniería Industrial - Concepción - Chile \\ elizepul@gmail.com
}

\begin{abstract}
Resumen
El objetivo del estudio fue evaluar la satisfacción de un segmento de estudiantes de niveles superiores de la carrera de ingeniería industrial de la Universidad el Bío-Bío, Chile, e identificar las dimensiones de calidad estudiantil de interés para enfocar de manera más eficaz el proceso de toma de decisiones y apoyar consecuentemente el proceso de acreditación de la citada carrera. La metodología utilizada fue el diseño y aplicación de una encuesta debidamente validada y la aplicación de la técnica de análisis de factores. Los principales resultados permitieron conocer objetivamente el nivel de satisfacción actual de los estudiantes bajo estudio así como aquellas dimensiones de calidad más sensibles. Luego de la aplicación del instrumento de medición se logró un alto índice de confiabilidad y validez, con un Alpha de Cronbach de 0,94 por lo que el instrumento puede ser utilizado en cualquier institución de educación superior que imparta la carrera de ingeniería industrial. Los resultados indican un nivel de satisfacción intermedio dentro de la escala de medición utilizada y las seis dimensiones relevantes para enfocar el proceso de toma de decisiones para la carrera en cuestión fueron las siguientes: Gestión Metodológica, Desarrollo personal, Servicios tecnológicos, Seguridad emocional, Seguridad fisica y Reconocimiento del éxito personal.
\end{abstract}

Palabras claves: análisis de factores, dimensiones de calidad estudiantil, ingeniería industrial, satisfacción de estudiantes.

\section{Introducción}

Es sabido que el quehacer universitario no está al margen de los desafíos que se le presentan para demostrar excelencia académica y administrativa ante sus clientes, así como lo demuestran las organizaciones que exigen cada día más de demostraciones de su compromiso con la calidad, la 
tendencias, por ejemplo, sobre organizaciones que año a año van adoptando estándares de calidad como ISO 9000, entre otros, sistemas de calidad presentes en el mercado actual.

En el ámbito universitario, antiguamente la calidad de la educación era medida a través de factores como infraestructura, calificación de los docentes y otras ventajas comparativas en relación a la formación académica. Hoy en día, es necesario comprometerse de manera más explícita con buenas prácticas de gestión y comprometerse con los principios modernos de calidad mundialmente conocidos (Gutiérrez, 2005), en este sentido, las universidades y carreras se ven enfrentados a la necesidad de medir y evaluar la calidad de los servicios, en particular reconocer el rol que juega la satisfacción de los estudiantes como determinante de la calidad de los servicios académicos que se les ofrece (Palacios, 2002). En el ámbito nacional, las universidades se están viendo forzadas a enfrentar la adopción de sistemas de calidad entendidos, en particular, como la adopción de prácticas que le permitan mantener vigentes su acreditación en los ámbitos del quehacer académico que declaran ante el mercado. Es así, en un mercado de competitividad en el contexto académico que las universidades y, en particular la carrera de ingeniería industrial, de la UBB, está comprometida a mantener y mejorar su proceso de acreditación, como evidencia que está comprometida con la calidad de los servicios que ofrece a sus estudiantes. De modo que tanto a nivel institucional como a nivel de carrera, ya no es extraño que deban reflejar, ambas organizaciones buenas prácticas de gestión ante sus educandos.

El contexto en que se desarrolla este trabajo está referido a la carrera de ingeniería civil industrial, de la Universidad del Bío Bío, de Chile (UBB), y en particular está orientada a un espectro de estudiantes de nivel medio y avanzado de la carrera, con la finalidad de poder focalizar los resultados del estudio dada la heterogeneidad de expectativas que se han detectado según avanzan los estudiantes en el logro de su título profesional.

En este afán por mejorar los procesos de toma de decisiones al interior del departamento de ingeniería industrial que administra la carrera de Carrera de Ingeniería Civil Industrial (ICI),se define el objetivo general de este estudio en el sentido "Conocer las dimensiones de calidad de mayor interés por parte de los estudiantes ICI, de la Universidad del Bío Bío”.

Dentro de las razones que justifican este trabajo investigativo se encuentra la necesidad de mejorar el proceso de toma de decisiones en el Departamento de Ingeniería Industrial y también incrementar las ventajas competitivas de la carrera en el ámbito regional, puesto que en la Región, en la última década, se ha experimentado un aumento tanto en la cantidad de instituciones universitarias que compiten por el mercado total, pasado de cuatro a quince universidades, y en el caso de la cantidad de carreras de ICI presentes actualmente en el mercado, éstas han aumentado de dos a cinco carreras en los últimos 10 años. En consecuencia, las evidencias indican que se deben atender las expectativas, intereses y necesidades de un grupo de estudiantes, (Pérez, 2000) para 
mantener la competitividad en el mercado.

Sobre las técnicas investigadas para poder analizar y determinar dimensiones de calidad con rigor científico se destacan los aportes identificados y descritos en Martínez y Mejías (2007), contribución que está también orientado para una carrera de ingeniería industrial, en la universidad de Carabobo, de Venezuela. Por otra parte, de recogen y analizan otros aportes como producto de aplicar similares metodologías pero desarrolladas en el contexto industrial y de servicios y no en el ámbito universitario, que sin embargo ofrecen nuevas perspectivas de análisis para tener en cuenta, entre ellos se destacan los aportes encontrados en: Garza y Pérez (2007), Barrios y Mejías (2007), Acosta y Mejías (2007), Siado y Mejías (2007), Manrique y Mejías (2009) y Blanco, Rivero y Maneiro (2009).

En particular la técnica multivariada utilizada en este estudio fue el Análisis de Factores debido que esta técnica permite reconocer y reducir las dimensiones de calidad cuando estas son numerosas y no se identifican claramente, aspecto central de los objetivos de este estudio.

Por otra parte, se seleccionaron estudiantes de la carrera de Ingeniería Civil Industrial que tuvieran un conocimiento más acabado de la situación actual de la carrera, tanto de la vida universitaria dentro y fuera del departamento, por este motivo la muestra de estudiantes seleccionada consideró aquellos estudiantes de cuarto año y superiores.

Pare efectos de obtención de datos de campo se aplicó una encuesta que consideró una exhaustiva revisión bibliográfica de los instrumentos ya validados en otras instituciones de educación superior adaptándose a la situación propia del Departamento de Ingeniería Industrial.

Los principales resultados permitieron identificar aquellas dimensiones de calidad que eran más valoradas por los estudiantes y en consecuencia poder utilizar esos resultados para enfocar de manera más efectiva el proceso de toma de decisiones en el ámbito de las actividades de difusión y marketing de la carrera.

\section{Materiales y métodos}

En el presente estudio la población objeto de la investigación estuvo constituida por estudiantes de cuarto año y niveles superiores lo que constituía un universos total de 303 estudiantes. La muestra fue seleccionada con un $95 \%$ de confianza y un $8 \%$ de error muestral requiriéndose en consecuencia un total de 100 estudiantes.

El instrumento de medición aplicado consistió en una encuesta que estaba dirigida a la muestra seleccionada. Para el diseño de la encuesta se realizó una exhaustiva revisión bibliográfica de los instrumentos ya validados en otras instituciones de educación superior (Garza y Pérez ,2007), así como revisión bibliográfica sobre la satisfacción estudiantil (ELLIOT; SHIN, 2002). Por otra 
parte, se consideró la experiencia de académicos en la aplicación de estudios similares, entre ellos los de (TORRES et al, 2004) de modo de analizar la validez de constructor y de contenido como se sugiere en Hernández (2006)en. Además, la encuesta se adaptó a la situación del Departamento de Ingeniería Industrial y se procedió a la aplicación de una prueba piloto, cuyo objetivo fue calcular la validez y confiabilidad de la encuesta aplicada.

Para el cálculo de la confiabilidad y validez se utilizó el Alpha de Cronbach, el cual es el método de fiabilidad más utilizado en psicometría. Se trata de un índice de consistencia interna que toma valores entre 0 y 1 y que sirve para comprobar si el instrumento que se está evaluando recopila información defectuosa y por tanto nos llevaría a conclusiones equivocadas. El coeficiente Alfa es por tanto un coeficiente de correlación al cuadrado que, a grandes rasgos, mide la homogeneidad de las preguntas promediando todas las correlaciones entre todos los ítems para ver que, si efectivamente, se parecen. Su interpretación será que, cuanto más se acerque el índice al extremo 1, mejor es la fiabilidad, considerando una fiabilidad respetable a partir de 0,80. En este estudio el índice en cuestión fue de 0.937 lo que nos indica una alta homogeneidad y equivalencia de respuesta a todos los ítems a la vez y para todos los encuestados, además, al eliminar elementos el Coeficiente Alfa sube sólo a 0,939, por lo tanto se desestimó el análisis de ítems, es decir, la posibilidad de eliminar uno o más ítems, debido a que es irrelevante el aumento experimentado en el índice en referencia.

La encuesta incluyó siete dimensiones teóricas que se indican en la columna Dimensión, de la Tabla 1. 
Tabla 1: Dimensiones teóricas e indicadores de la satisfacción estudiantil

\begin{tabular}{|c|c|c|c|}
\hline Dimensión & Definición & Indicador & Ítems \\
\hline $\begin{array}{l}\text { 1. Condiciones Básicas de } \\
\text { Infraestructura }\end{array}$ & $\begin{array}{l}\text { Apariencia y Calidad de las } \\
\text { instalaciones físicas para el } \\
\text { estudio el descanso y la } \\
\text { recreación }\end{array}$ & $\begin{array}{l}\text { - Limpieza de las instalaciones de estudio, } \\
\text { sanitarias y de descanso. } \\
\text { - Ventilación adecuada de las salas } \\
\text { - Comodidad del mobiliario } \\
\text { - Espacios para el estudio y descanso } \\
\text { - Infraestructura adecuada. }\end{array}$ & $1-10$ \\
\hline $\begin{array}{l}\text { 2. Servicios de la } \\
\text { Institución }\end{array}$ & $\begin{array}{l}\text { Satisfacción con respecto a los } \\
\text { servicios que brinda la } \\
\text { Universidad y el Departamento }\end{array}$ & $\begin{array}{l}\text { - Servicio bibliotecario, casino, servicio } \\
\text { médico y fotocopiado } \\
\text { - Proceso de inscripción de asignaturas } \\
\text { expedito } \\
\text { - Apoyo de federación y centro de } \\
\text { estudiantes } \\
\text { - Acceso a información y tecnologías de } \\
\text { información }\end{array}$ & $11-22$ \\
\hline $\begin{array}{l}\text { 3. Condiciones de } \\
\text { Seguridad }\end{array}$ & $\begin{array}{l}\text { Satisfacción con respecto a las } \\
\text { condiciones de seguridad que } \\
\text { brinda tanto el Departamento } \\
\text { como la Universidad }\end{array}$ & $\begin{array}{l}\text { - Salidas de emergencia de las } \\
\text { dependencias } \\
\text { - Mobiliario seguro } \\
\text { - Sensación de seguridad dentro y fuera de } \\
\text { la Universidad }\end{array}$ & $23-27$ \\
\hline 4. Trato afectuoso & $\begin{array}{l}\text { Satisfacción por la seguridad } \\
\text { emocional y trato por parte de } \\
\text { funcionarios }\end{array}$ & $\begin{array}{l}\text { - Trato afectuoso del personal de } \\
\text { biblioteca, servicio médico. } \\
\text { - Trato de funcionarios y docentes del } \\
\text { Departamento de Ingeniería Industrial }\end{array}$ & $28-34$ \\
\hline $\begin{array}{l}\text { 5. Proceso de enseñanza y } \\
\text { aprendizaje }\end{array}$ & $\begin{array}{llr}\text { Satisfacción } & \text { con } & \text { la } \\
\text { metodologías, } & \text { técnicas } & \text { y } \\
\text { evaluación por parte de } & \text { los } \\
\text { docentes } & & \end{array}$ & $\begin{array}{l}\text { - Malla curricular actualizada } \\
\text { - Incorporación de nuevas tecnologías a las } \\
\text { clases } \\
\text { - Sistema de evaluación } \\
\text { - Preparación, actualización y condiciones } \\
\text { pedagógicas de los docentes }\end{array}$ & $35-44$ \\
\hline 6. Logros Personales & $\begin{array}{l}\text { Satisfacción por los logros } \\
\text { personales obtenidos durante la } \\
\text { formación en el Departamento }\end{array}$ & $\begin{array}{l}\text { - Calificaciones obtenidas } \\
\text { - Dominio de contenido y técnicas de } \\
\text { estudio } \\
\text { - Desarrollo integral de la persona }\end{array}$ & $45-49$ \\
\hline $\begin{array}{l}\text { 7. Reconocimiento del } \\
\text { éxito personal }\end{array}$ & $\begin{array}{l}\text { Satisfacción por sentir } \\
\text { reconocimiento por parte de la } \\
\text { Universidad, Departamento y } \\
\text { compañeros }\end{array}$ & $\begin{array}{l}\text { - Reconocimiento de los logros por parte } \\
\text { de la Universidad, Departamento y } \\
\text { compañeros. }\end{array}$ & $50-52$ \\
\hline
\end{tabular}

Fuente: Elaboración propia (2009)

Además, la encuesta incluyó una portada, a modo de presentación, además, se incluyó una explicación del objetivo de la encuesta, permitiendo recopilar datos de criterio de clasificación, que sirvieron para posteriores análisis.

La posibilidad de respuesta para cada uno de los 52 ítems se presentó en una escala de tipo Likert, donde el estudiante debió circunscribirse a la elección de 5 opciones, del uno al cinco, donde el uno representa una condición de totalmente insatisfecho y el número cinco representa la condición de muy satisfecho

Para determinar el nivel de satisfacción que tiene el estudiantado según sus respuestas, se desarrolló una tabla (Ver tabla 2), en la cuál se aprecia el nivel alcanzado de satisfacción según su puntaje en las distintas dimensiones teóricas planteadas en la encuesta y en su nivel de satisfacción total (puntaje total). Los pares de valores que se presentan en la Tabla 2 representan la cantidad 
mínima y máxima de puntaje que es posible obtener en cada ítem de preguntas y en cada tramo de respuesta.

Tabla 2: Escala de valoración del cuestionario

\begin{tabular}{|c|c|c|c|c|c|}
\hline \multirow[b]{2}{*}{ Dimensión } & \multicolumn{5}{|c|}{ NIVEL DE SATISFACCION } \\
\hline & $\begin{array}{l}\text { Totalmente } \\
\text { Insatisfecho } \\
\text { (1) }\end{array}$ & $\begin{array}{c}\text { Poco } \\
\text { Satisfecho } \\
\quad(2)\end{array}$ & $\begin{array}{c}\text { Satisfecho } \\
\text { (3) }\end{array}$ & $\begin{array}{c}\text { Bastante } \\
\text { Satisfecho } \\
\text { (4) }\end{array}$ & $\begin{array}{c}\text { Muy } \\
\text { Satisfecho } \\
\text { (5) }\end{array}$ \\
\hline $\begin{array}{l}\text { SATISFACCION TOTAL } \\
\text { 1. Satisfacción por las } \\
\text { condiciones básicas de } \\
\text { infraestructura: }\end{array}$ & $1-52$ & $53-104$ & $105-156$ & $157-208$ & $209-260$ \\
\hline 1.1 En la Universidad & $1-3$ & $4-6$ & $7-9$ & $10-12$ & $13-15$ \\
\hline 1.2 En el departamento & $1-7$ & $8-14$ & $15-21$ & $22-28$ & $29-35$ \\
\hline $\begin{array}{l}\text { 2. Satisfacción por los } \\
\text { servicios prestados: }\end{array}$ & $1-12$ & $13-24$ & $25-36$ & $37-48$ & $49-60$ \\
\hline 2.1 Por el Departamento & $1-5$ & $6-10$ & $11-15$ & $16-20$ & $21-25$ \\
\hline 2.2 Por la Universidad & $1-7$ & $8-14$ & $15-21$ & $22-28$ & $29-35$ \\
\hline $\begin{array}{l}\text { 3. Satisfacción por las } \\
\text { condiciones de seguridad: }\end{array}$ & $1-5$ & $6-10$ & $11-15$ & $16-20$ & $21-25$ \\
\hline 3.1 En el Departamento & $1-2$ & $3-4$ & $5-6$ & $7-8$ & $9-10$ \\
\hline 3.2 En la Universidad & $1-3$ & $4-6$ & $7-9$ & $10-12$ & $13-15$ \\
\hline $\begin{array}{l}\text { 4. Satisfacción por el trato } \\
\text { afectuoso del personal: }\end{array}$ & $1-7$ & $8-14$ & $15-21$ & $22-28$ & $29-35$ \\
\hline 4.1 En al Departamento & $1-5$ & $6-10$ & $11-15$ & $16-20$ & $21-25$ \\
\hline 4.2 En la Universidad & $1-2$ & $3-4$ & $5-6$ & $7-8$ & $9-10$ \\
\hline $\begin{array}{l}\text { 5. Satisfacción por el proceso } \\
\text { de enseñanza y aprendizaje }\end{array}$ & $1-10$ & $11-20$ & $21-30$ & $31-40$ & $41-50$ \\
\hline $\begin{array}{l}\text { 6. Satisfacción por los logros } \\
\text { personales }\end{array}$ & $1-5$ & $6-10$ & $11-15$ & $16-20$ & $21-25$ \\
\hline $\begin{array}{l}\text { 7. Satisfacción por el } \\
\text { reconocimiento del éxito } \\
\text { personal }\end{array}$ & $1-3$ & $4-6$ & $7-9$ & $10-12$ & $13-15$ \\
\hline
\end{tabular}

Fuente: Elaboración propia (2009)

Debido al elevado número de variables (52), se empleó la técnica de análisis factorial dado su valor psicométrico para validar cuestionarios, así como su capacidad para estudiar las relaciones entre las variables propuestas y advertir una estructura dimensional entre ellas. Para el análisis de datos se conformó una matriz de orden $89 \times 52$ para los estudiantes encuestados, donde el orden de las matrices corresponde al número de encuestas respondidas en su totalidad y el número de ítemes o preguntas contenidas en la encuesta. Las encuestas con datos faltantes no se analizaron, cabe señalar que las encuestas con datos faltantes, correspondieron a un total de 11, las cuales en su mayoría pertenecían a estudiantes que se detectó que no tenían un conocimiento profundo acerca de servicios que prestaba la institución, como por ejemplo, conocimientos sobre las características del servicio de saludtransformándose la población objetivo inicial en el conjunto de estudiantes que efectivamente conocen los servicios y en consecuencia estimándose necesario la eliminación de aquellas encuestas con datos faltantes. 
Para que el análisis de factores fuese correcto era necesario verificar previamente que las variables tenían una buena correlación entre sí, por lo que se realizó un análisis de la matriz de correlaciones calculada a partir de los datos obtenidos en la encuesta y determinar la adecuación del uso de este análisis como se sugiere en Prat y Doval (2003). Se entenderá como buena correlación a aquellos valores que presenten in índice de correlación igual o superior a 0.5.

Para el análisis de las dimensiones se utilizó como herramienta estadística el análisis de componentes principales con rotación varimax. Este procedimiento estadístico facilita el análisis y la interpretación de los datos garantizando la menor pérdida de información posible y consiste en la obtención de un número reducido de factores que permite explicar la mayor parte de la variabilidad total de las variables observadas. El porcentaje de variabilidad que explica cada uno de los factores suele ser diferente, siendo unos pocos factores los que explican gran parte de la variabilidad total. El análisis de componentes principales es un método 'blando' al no requerir hipótesis previas sobre la estructura de los datos y sus interrelaciones, ni sobre la distribución de probabilidad multivariante a la que responden. No obstante, es un método que permite averiguar, aunque de forma exploratoria, en qué medida las dimensiones que se obtienen coinciden con las dimensiones teóricas que se vislumbran en la literatura.

Como paso previo a la aplicación del método de componentes principales con rotación varimax a los diferentes constructos fue necesario analizar la idoneidad estadística utilizándose para ello la prueba de esfericidad de Bartlett y la medida de Kaiser-Meyer-Olkin. Concretamente, el test de esfericidad de Bartlett permite contrastar la hipótesis nula de que la matriz de correlaciones es una matriz identidad, cuya aceptación implicaría el replanteamiento de la utilización del análisis de componentes principales. Por su parte, la medida de adecuación de Kaiser-Meyer-Olkin (o índice KMO) es útil para comparar los valores de los coeficientes de correlación observados con los coeficientes de correlación parcial, de tal forma que valores pequeños indican que el análisis de componentes principales no es aconsejable, se recomienda como límite de aceptación de este índice KMO valores superiores a 0,5 (Pardo y Ruiz, 2002)

Con la totalidad de datos validados, de acuerdo a lo señalado en Johnson (2000), utilizamos la rotación varimax que hace más simple la interpretación de los componentes y la determinación de las relaciones existentes entre los factores y las variables en estudio. El procedimiento utilizado para simplificar la interpretación de los factores está dada por:

a) Comenzando con la primera variable y el primer factor, y moviéndose horizontalmente de izquierda a derecha a través de los módulos, ennegrecer las cargas con mayor magnitud en valor absoluto. Luego, considerar la segunda variable, y nuevamente moverse de izquierda a derecha en 
forma horizontal, identificando las mayores cargas en valor absoluto y ennegreciéndolas. Continuar el proceso para cada variable.

b) Una vez que todas las variables fueron consideradas, se examina cada valor en negrita para determinar si una carga es "significativa". Esta valoración puede ser hecha sobre la base de la significancia estadística del coeficiente de correlación (carga). Para que esto se cumpla, el factor solución debe explicar a lo menos el 50\% de la varianza de la variable. Esto se refiere a que la carga más pequeña debe ser superior a 0.54 (valor absoluto), para ser considerada significativa. Los valores que cumplan esta condición son subrayados.

c) Examinar la matriz de rotación varimax para identificar las variables que no han sido subrayadas y, por tanto, no cargan en ningún factor. El investigador, entonces, ha de decidir si el resto del análisis se efectúa sobre aquellas variables consideradas significativas o evalúa, críticamente, cada variable en términos de su importancia y su comunalidad.

d) Basados en los resultados del paso anterior, se debe dar un significado a los distintos factores. Las variables con altas cargas son consideradas de gran influencia. Para el procesamiento de la información se utilizó el software estadístico SPSS para Windows, V12.0.

\section{Resultados}

En relación a los resultados de las encuestas, estos se abordaron en base a cuatro criterios:

- Índice de satisfacción según dimensiones teóricas: Compuesto por las siete dimensiones planteadas en la elaboración del cuestionario.

- Satisfacción según año de ingreso, edad y género

- Índices de valoración: Constituido por los mayores y menores puntajes clasificados según los ítems de la encuesta.

- Índice de Satisfacción Total: Conformado por el nivel de satisfacción total de los encuestados.

\section{1 Índice de satisfacción según dimensiones teóricas}

El primer resultado de este estudio fue el índice de satisfacción según las dimensiones teóricas, como se presenta en la Tabla 3. Este índice nos indica el grado de satisfacción que tienen los estudiantes sobre cada una de las dimensiones de calidad teóricas incluidas en el cuestionario. El instrumento de medición permitió discriminar entre satisfacción respecto del Departamento como de la Universidad pero solo para las cuatro primeras dimensiones de estudio. Los resultados 
obtenidos no permitieron observar diferencias cualitativas evidentes entre Departamento y Universidad, como inicialmente así se presumía.

El nivel de satisfacción total obtenido fue de "Satisfecho", es decir, un nivel de evaluación de 3 , en la escala de 1 a 5 .

Tabla 3: Nivel de satisfacción según dimensiones teóricas

\begin{tabular}{|c|c|c|}
\hline Dimensión & Puntuación & Nivel de Satisfacción \\
\hline $\begin{array}{l}\text { 1. Satisfacción por las condiciones } \\
\text { básicas de infraestructura }\end{array}$ & 24 & $\mathrm{~S}$ \\
\hline 1.1 En la universidad & 8 & S \\
\hline 1.2 En el departamento & 16 & S \\
\hline $\begin{array}{l}\text { 2. Satisfacción por los servicios } \\
\text { prestados }\end{array}$ & 34 & $\mathrm{~S}$ \\
\hline 2.1 Por el Departamento & 15 & S \\
\hline 2.2 Por la Universidad & 19 & S \\
\hline $\begin{array}{l}\text { 3. Satisfacción por las condiciones } \\
\text { de seguridad }\end{array}$ & 13 & S \\
\hline 3.1 En el Departamento & 5 & PS \\
\hline 3.2 En la Universidad & 8 & $\mathrm{~S}$ \\
\hline $\begin{array}{l}\text { 4. Satisfacción por el trato afectuoso } \\
\text { del personal: }\end{array}$ & 24 & BS \\
\hline 4.1 En al Departamento & 17 & BS \\
\hline 4.2 En la Universidad & 7 & BS \\
\hline $\begin{array}{l}\text { 5. Satisfacción por el proceso de } \\
\text { enseñanza y aprendizaje }\end{array}$ & 32 & BS \\
\hline $\begin{array}{l}\text { 6. Satisfacción por los logros } \\
\text { personales }\end{array}$ & 15 & $\mathrm{~S}$ \\
\hline $\begin{array}{l}\text { 7. Satisfacción por el } \\
\text { reconocimiento del éxito personal }\end{array}$ & 8 & S \\
\hline SATISFACCIÓN TOTAL & 150 & $\mathrm{~S}$ \\
\hline
\end{tabular}

Nota: S: indica "Satisfecho"; BS: indica "Bastante Satisfecho"; PS: indica "Poco Satisfecho" Fuente: Elaboración propia (2009)

\subsection{Satisfacción según año de ingreso, edad y género}

En segunda instancia se procedió a disgregar los datos según año de ingreso, edad y género (Tablas 4 y 5). En cuanto al año de ingreso se observó que a mayor cantidad de años de permanencia en el Departamento, mayor es el nivel de satisfacción observado por parte de los estudiantes. En cuanto a la edad de los estudiantes, no se observó una tendencia en particular. Finalmente, en cuanto a la variable género, se puede revelar que las mujeres muestran un índice de satisfacción mayor que los hombres, ya que ellas declaran estar "bastante satisfechas" en relación a los hombres que solo dicen estar "satisfechos". 
Tabla 4: Nivel de satisfacción según año de ingreso

\begin{tabular}{lcccc}
\hline $\begin{array}{l}\text { Año de } \\
\text { Ingreso }\end{array}$ & $\begin{array}{c}\text { Cantidad de } \\
\text { Estudiantes }\end{array}$ & $\begin{array}{c}\text { Años en la } \\
\text { Universidad }\end{array}$ & $\begin{array}{c}\text { Puntuación } \\
\text { media }\end{array}$ & $\begin{array}{c}\text { Nivel de } \\
\text { Satisfacción }\end{array}$ \\
\hline 2001 & 4 & 9 & 168 & $\mathrm{BS}$ \\
2002 & 4 & 8 & 158 & $\mathrm{BS}$ \\
2003 & 18 & 7 & 165 & $\mathrm{BS}$ \\
2004 & 19 & 6 & 156 & $\mathrm{BS}$ \\
2005 & 39 & 5 & 145 & $\mathrm{~S}$ \\
2006 & 5 & 4 & 132 & $\mathrm{~S}$ \\
\hline Fuente· Elaboración propia (2009) & & &
\end{tabular}

Tabla 5: Nivel de satisfacción según año de ingreso

\begin{tabular}{ccc}
\hline $\begin{array}{c}\text { Edad } \\
\text { estudiantes } \\
\text { (años) }\end{array}$ & $\begin{array}{c}\text { Cantidad de } \\
\text { Estudiantes }\end{array}$ & $\begin{array}{c}\text { Nivel de } \\
\text { Satisfacción }\end{array}$ \\
\hline 21 & 1 & $\mathrm{~S}$ \\
22 & 25 & $\mathrm{~S}$ \\
23 & 28 & $\mathrm{~S}$ \\
24 & 14 & $\mathrm{BS}$ \\
25 & 9 & $\mathrm{BS}$ \\
26 & 7 & $\mathrm{BS}$ \\
27 & 3 & $\mathrm{~S}$ \\
28 & 2 & $\mathrm{~S}$ \\
\hline Fuente: Elaboración propia (2009) &
\end{tabular}

\section{3 Índices de valoración}

En tercer lugar se identificaron los ítemes con menores y mayores valoraciones por parte de los estudiantes como se presentan en las Tablas 6 y 7. De los resultados se observa, por una parte, que en ninguna de los ítemes investigados se alcanzó el puntaje mínimo ni el puntaje máximo. Sin embargo, se puede mencionar que los puntajes más bajos están relacionados con los ítems de la dimensión Condiciones básicas de Infraestructura en cambio, los ítems mejor valorados se relacionan con los ítems de la dimensión Trato Afectuoso por parte de los académicos y administrativos del departamento de ingeniería industrial. 
Tabla 6: Menores índices de satisfacción estudiantil por ítem

\begin{tabular}{|c|c|c|c|c|}
\hline $\begin{array}{l}\mathbf{N}^{0} \\
\text { Ittem }\end{array}$ & Descripción & $\begin{array}{l}\text { Dimensión a la que } \\
\text { pertenece }\end{array}$ & Valoración & Leyenda \\
\hline 2 & $\begin{array}{l}\text { Ventilación de las aulas del Departamento de } \\
\text { Ingeniería Industrial }\end{array}$ & $\begin{array}{l}\text { Condiciones básicas de } \\
\text { Infraestructura }\end{array}$ & 2 & PS \\
\hline 3 & $\begin{array}{l}\text { Comodidad del mobiliario del Departamento de } \\
\text { Ingeniería Industrial }\end{array}$ & $\begin{array}{l}\text { Condiciones básicas de } \\
\text { Infraestructura }\end{array}$ & 2 & PS \\
\hline 4 & $\begin{array}{l}\text { Instalaciones adecuadas para la cantidad de estudiantes } \\
\text { en el Departamento de Ingeniería Industrial }\end{array}$ & $\begin{array}{l}\text { Condiciones básicas de } \\
\text { Infraestructura }\end{array}$ & 2 & PS \\
\hline 5 & $\begin{array}{l}\text { Espacios para el estudio en el Departamento de } \\
\text { Ingeniería Industrial }\end{array}$ & $\begin{array}{l}\text { Condiciones básicas de } \\
\text { Infraestructura }\end{array}$ & 2 & PS \\
\hline 6 & $\begin{array}{l}\text { Instalaciones sanitarias adecuadas y limpias dentro del } \\
\text { Departamento de Ingeniería Industrial }\end{array}$ & $\begin{array}{l}\text { Condiciones básicas de } \\
\text { Infraestructura }\end{array}$ & 2 & PS \\
\hline 7 & $\begin{array}{l}\text { Espacios para el descanso y recreación dentro del } \\
\text { Departamento de Ingeniería Industrial }\end{array}$ & $\begin{array}{l}\text { Condiciones básicas de } \\
\text { Infraestructura }\end{array}$ & 2 & PS \\
\hline 14 & Servicio de Fotocopiado & $\begin{array}{l}\text { Servicios de la Institución } \\
\text { (Universidad) }\end{array}$ & 2 & PS \\
\hline 17 & El apoyo que brinda la federación estudiantil & $\begin{array}{l}\text { Servicios de la Institución } \\
\text { (Universidad) }\end{array}$ & 2 & PS \\
\hline 26 & $\begin{array}{l}\text { Condiciones de seguridad en el Departamento de } \\
\text { Ingeniería Industrial }\end{array}$ & Condiciones de Seguridad & 2 & PS \\
\hline 32 & Trato afectuoso de la Jefa de carrera & Trato afectuoso & 2 & PS \\
\hline 52 & $\begin{array}{l}\text { Reconocimiento del éxito personal por parte de los } \\
\text { compañeros }\end{array}$ & $\begin{array}{l}\text { Reconocimiento del éxito } \\
\text { personal }\end{array}$ & 2 & PS \\
\hline
\end{tabular}

Fuente: Elaboración propia (2009)

Tabla 7: Mayores índices de satisfacción estudiantil por ítem

\begin{tabular}{|c|c|c|c|c|}
\hline $\mathbf{N}^{0}$ Ítem & Descripción & $\begin{array}{l}\text { Dimensión a la que } \\
\text { pertenece }\end{array}$ & Valoración & Leyenda \\
\hline 11 & Servicio Bibliotecario & $\begin{array}{l}\text { Servicios de la } \\
\text { Institución }\end{array}$ & 4 & $\mathrm{BS}$ \\
\hline 19 & $\begin{array}{l}\text { Acceso a computadores en el Departamento de } \\
\text { Ingeniería Industrial }\end{array}$ & $\begin{array}{l}\text { Servicios de la } \\
\text { Institución } \\
\text { (Departamento) }\end{array}$ & 4 & $\mathrm{BS}$ \\
\hline 20 & $\begin{array}{l}\text { Acceso a internet y correo electrónico en el } \\
\text { Departamento de Ingeniería Industrial }\end{array}$ & $\begin{array}{l}\text { Servicios de la } \\
\text { Institución } \\
\text { (Departamento) }\end{array}$ & 4 & $\mathrm{BS}$ \\
\hline 28 & Trato afectuoso del personal de biblioteca & Trato afectuoso & 4 & $\mathrm{BS}$ \\
\hline 31 & $\begin{array}{l}\text { Trato afectuoso del director del Departamento de } \\
\text { Ingeniería Industrial }\end{array}$ & Trato afectuoso & 4 & $\mathrm{BS}$ \\
\hline 33 & $\begin{array}{l}\text { Trato afectuoso de las secretarias y auxiliar del } \\
\text { Departamento de Ingeniería Industrial }\end{array}$ & Trato afectuoso & 4 & $\mathrm{BS}$ \\
\hline 34 & $\begin{array}{l}\text { Trato afectuoso del personal de apoyo del } \\
\text { laboratorio de computación del Departamento de } \\
\text { Ingeniería Industrial }\end{array}$ & Trato afectuoso & 4 & BS \\
\hline 44 & Incorporación de tecnología para las clases & $\begin{array}{l}\text { Proceso de enseñanza } \\
\text { y aprendizaje }\end{array}$ & 4 & $\mathrm{BS}$ \\
\hline
\end{tabular}

Fuente: Elaboración propia (2009)

\section{4 Índice de Satisfacción Total}

Finalmente, los resultados asociados con el nivel de satisfacción total se destaca que no existen estudiantes "Totalmente Insatisfechos", a su vez, solo el 1\% de los estudiantes señala estar "Muy Satisfecho" y la mayoría (90\% de los estudiantes) presentan una valoración entre Satisfecho y Bastante Satisfecho. La visualización de los perfiles de respuesta se indican en la Figura 1. 
Figura 1: Nivel de satisfacción total

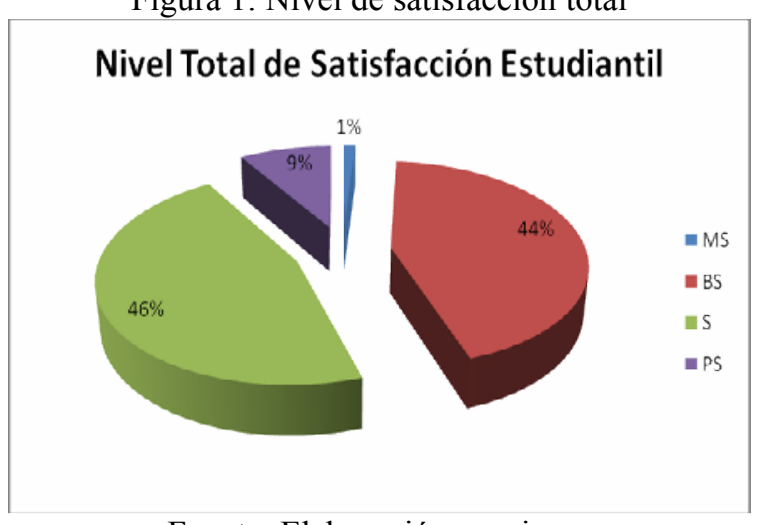

Fuente: Elaboración propia

En relación con los resultados de la aplicación del análisis de factores, estos se presentan en las tablas 8, 9 y 10, en términos del número de componentes, valores propios y porcentajes de varianza, además de las comunalidades y la matriz de rotación varimax.

Tabla 8: Porcentaje de varianza explicada

\begin{tabular}{|c|c|c|c|c|c|c|c|c|c|}
\hline \multirow[t]{2}{*}{ Componente } & \multicolumn{3}{|c|}{ Autovalores iniciales } & \multicolumn{3}{|c|}{$\begin{array}{l}\text { Sumas de las saturaciones } \\
\text { al cuadrado de la } \\
\text { extracción }\end{array}$} & \multicolumn{3}{|c|}{$\begin{array}{l}\text { Suma de las saturaciones } \\
\text { al cuadrado de la rotación }\end{array}$} \\
\hline & Total & $\begin{array}{l}\% \text { de la } \\
\text { varianza }\end{array}$ & $\begin{array}{l}\% \\
\text { acumulado }\end{array}$ & Total & $\begin{array}{l}\% \text { de la } \\
\text { varianza }\end{array}$ & $\begin{array}{l}\% \\
\text { acumulado }\end{array}$ & Total & $\begin{array}{l}\% \text { de la } \\
\text { varianza }\end{array}$ & $\begin{array}{l}\% \\
\text { acumulado }\end{array}$ \\
\hline 1 & 7,807 & 37,175 & 37,175 & 7,807 & 37,175 & 37,175 & 3,926 & 18,698 & 18,698 \\
\hline 2 & 1,633 & 7,777 & 44,952 & 1,633 & 7,777 & 44,952 & 3,294 & 15,683 & 34,381 \\
\hline 3 & 1,564 & 7,447 & 52,399 & 1,564 & 7,447 & 52,399 & 2,174 & 10,352 & 44,733 \\
\hline 4 & 1,488 & 7,088 & 59,487 & 1,488 & 7,088 & 59,487 & 2,085 & 9,928 & 54,661 \\
\hline 5 & 1,306 & 6,220 & 65,706 & 1,306 & 6,220 & 65,706 & 1,703 & 8,110 & 62,771 \\
\hline 6 & 1,059 & 5,044 & 70,751 & 1,059 & 5,044 & 70,751 & 1,676 & 7,979 & 70,751 \\
\hline
\end{tabular}

Fuente: Elaboración propia según resultados SPSS (2009)

Tabla 9: Comunalidades

\begin{tabular}{|c|c|c|}
\hline \multicolumn{2}{|c|}{$\mathbf{N}^{0}$} & \multirow[b]{2}{*}{ Extracción } \\
\hline Item & Inicial & \\
\hline Item19 & 1,000 & 0,886 \\
\hline Item 20 & 1,000 & 0,835 \\
\hline Item 23 & 1,000 & 0,796 \\
\hline Item 26 & 1,000 & 0,794 \\
\hline Item 30 & 1,000 & 0,757 \\
\hline Item33 & 1,000 & 0,658 \\
\hline Item34 & 1,000 & 0,739 \\
\hline Item35 & 1,000 & 0,587 \\
\hline Item36 & 1,000 & 0,623 \\
\hline Item37 & 1,000 & 0,688 \\
\hline Item38 & 1,000 & 0,583 \\
\hline Item39 & 1,000 & 0,609 \\
\hline Item 40 & 1,000 & 0,630 \\
\hline Item41 & 1,000 & 0,731 \\
\hline Item45 & 1,000 & 0,531 \\
\hline Item46 & 1,000 & 0,618 \\
\hline Item47 & 1,000 & 0,739 \\
\hline Item 48 & 1,000 & 0,689 \\
\hline Item49 & 1,000 & 0,723 \\
\hline Item50 & 1,000 & 0,822 \\
\hline Item51 & 1,000 & 0,820 \\
\hline
\end{tabular}


Tabla 10: Matriz de componentes rotados

\begin{tabular}{|c|c|c|c|c|c|c|}
\hline \multirow{2}{*}{ Ítem } & \multicolumn{6}{|c|}{ Componente } \\
\hline & 1 & 2 & 3 & 4 & 5 & 6 \\
\hline Item37 & 0,755 & & & & & \\
\hline Item 40 & 0,736 & & & & & \\
\hline Item39 & 0,706 & & & & & \\
\hline Item38 & 0,686 & & & & & \\
\hline Item41 & 0,668 & & & & & \\
\hline Item36 & 0,642 & & & & & \\
\hline Item35 & 0,502 & & & & & \\
\hline Item47 & & 0,796 & & & & \\
\hline Item49 & & 0,741 & & & & \\
\hline Item48 & & 0,714 & & & & \\
\hline Item46 & & 0,686 & & & & \\
\hline Item 45 & & 0,554 & & & & \\
\hline Item19 & & & 0,910 & & & \\
\hline Item 20 & & & 0,864 & & & \\
\hline Item34 & & & & 0,841 & & \\
\hline Item33 & & & & 0,730 & & \\
\hline Item30 & & & & 0,632 & & \\
\hline Item 23 & & & & & 0,875 & \\
\hline Item 26 & & & & & 0,851 & \\
\hline Item50 & & & & & & 0,882 \\
\hline Item51 & & & & & & 0.822 \\
\hline
\end{tabular}

Fuente: Elaboración propia según resultados SPSS (2009)

Siguiendo con la metodología descrita anteriormente, se logró seleccionar inicialmente 27 variables que cumplían con una correlación superior a 0.5 , al realizar el análisis factorial, se obtuvieron 7 factores que explicaban el $68,749 \%$ de la varianza. A continuación se eliminaron del análisis los ítems cuya carga factorial resultó inferior a 0,49, seguidamente se rehízo el análisis con los 23 ítems restantes y se obtuvo una solución factorial que explicaba el 70,3\% de la varianza total, no obstante los ítems 7 y 18 no alcanzaron una carga factorial superior a 0,49 , por lo que fueron eliminados. Se repitió el análisis factorial, esta vez con los 21 ítems y se obtuvo 6 factores que explicaban el 70,7\% de la varianza total por lo tanto se trabajó con esta solución.

Para el caso del indicador KMO éste debe tomar un valor aproximado a 1 ya que los valores por debajo de 0,6 son considerados insatisfactorios para utilizar el análisis de factores con esos datos, en este caso, el indicador dio un resultado de 0,794 considerado bueno (Pardo Y Ruiz, 2002).

El estudio de las comunalidades presenta valores mayores que 0.53 , estos valores corresponden a la proporción de la varianza de la variable que es explicada por los factores. El valor del determinante resultó ser 3,05E-06 lo cual nos indicó que las variables están linealmente correlacionadas al aproximarse al valor cero, lo cual es un buen indicador de la idoneidad del análisis.

En la Tabla 10 se observan las estimaciones de los coeficientes de las variables utilizando 
rotación varimax para lograr una mejor interpretación, con estos datos en la Tabla 11 se observan las seis dimensiones obtenidas a partir del análisis de factores.

Tabla 11 Dimensiones de satisfacción estudiantil

\begin{tabular}{|c|c|}
\hline Dimensión & Dimensiones de calidad \\
\hline \multirow[t]{9}{*}{1} & GESTION METODOLOGICA DE LA CARRERA \\
\hline & 35. Flujo, créditos y asignaturas de la malla de la carrera Ingeniería Industrial \\
\hline & 36. Contenido de las asignaturas de la carrera Ingeniería Civil Industrial \\
\hline & 37. Dominio de los contenidos de los profesores del Departamento de Ingeniería \\
\hline & 38. Nivel de exigencia de los profesores del Departamento de Ingeniería Industrial. \\
\hline & 39. Sistema de evaluación de las asignaturas del Departamento de Ingeniería \\
\hline & 40 Anovo de los profesores del Denartamento de Inoeniería Industrial en horarios de \\
\hline & $\begin{array}{l}\text { 40. Apoyo de los profesores del Departamento de Ingeniería Industrial en horarios de } \\
\text { consulta. }\end{array}$ \\
\hline & 41. Calidad adecuada del cuerpo docente del Departamento de Ingeniería Industrial. \\
\hline \multirow[t]{8}{*}{2} & DESARROLLO PERSONAL \\
\hline & 45. Conocimientos adquiridos en el Departamento de Ingeniería Industrial. \\
\hline & $\begin{array}{l}\text { 46. Calificaciones obtenidas en las asignaturas del Departamento de Ingeniería } \\
\text { Industrial }\end{array}$ \\
\hline & 47. Dominio de Técnicas de estudio y trabajo Intelectual inducido por el \\
\hline & Departamento de Ingeniería Industrial \\
\hline & 48. Fomento del trabajo en equipo inducido por el Departamento de Ingeniería \\
\hline & Industrial \\
\hline & $\begin{array}{l}\text { 49. Desarrollo integral de la persona inducido por el Departamento de Ingeniería } \\
\text { Industrial }\end{array}$ \\
\hline \multirow[t]{3}{*}{3} & SERVICIOS TECNOLOGICOS \\
\hline & $\begin{array}{l}\text { 19. Acceso a Internet y correo electrónico en el Departamento de Ingeniería } \\
\text { Industrial }\end{array}$ \\
\hline & 20. Acceso a computadores en el Departamento de Ingeniería Industrial \\
\hline \multirow[t]{4}{*}{4} & SEGURIDAD EMOCIONAL \\
\hline & 30. Trato afectuoso de los profesores del Departamento de Ingeniería Industrial. \\
\hline & $\begin{array}{l}\text { 33. Trato afectuoso de las secretarias y auxiliar del Departamento de Ingeniería } \\
\text { Industrial. }\end{array}$ \\
\hline & $\begin{array}{l}\text { 34. Trato afectuoso del personal de apoyo del laboratorio de computación del } \\
\text { Departamento de Ingeniería Industrial. }\end{array}$ \\
\hline \multirow[t]{3}{*}{5} & SEGURIDAD FISICA \\
\hline & 23. Condiciones de seguridad en la Universidad \\
\hline & 26. Condiciones de seguridad en el Departamento de Ingeniería Industrial \\
\hline \multirow[t]{3}{*}{6} & RECONOCIMIENTO DEL ÉXITO PERSONAL \\
\hline & 50. Reconocimiento del éxito personal por parte de la Universidad \\
\hline & 51. Reconocimiento del éxito personal por parte del Departamento Ingeniería \\
\hline
\end{tabular}

Fuente: Elaboración Propia (2009)

Para finalizar el análisis, se midió el nivel de satisfacción de los estudiantes en cada una de estas nuevas dimensiones, cuyo resumen se encuentra en la tabla 12.

Luego de analizar los resultados de la tabla 12, se observa que los estudiantes tienen para estas dimensiones un índice de "satisfecho" ó "bastante satisfecho" lo que se relacionaría directamente con que el 90\% de los estudiantes, presenta un nivel de satisfacción total superior a los tres puntos, que corresponde a "Satisfecho", ya que se sienten satisfechos en aquellas dimensiones que realmente les importa. 
Tabla 12: Nivel de Satisfacción según análisis de factores

\begin{tabular}{lcc}
\hline Dimensión & Puntuación & Nivel de Satisfacción \\
\hline Gestión metodológica de la carrera & 22 & $\mathrm{BS}$ \\
Desarrollo personal & 15 & $\mathrm{~S}$ \\
Servicios Tecnológicos & 8 & $\mathrm{BS}$ \\
Seguridad emocional & 11 & $\mathrm{BS}$ \\
Seguridad física & 5 & $\mathrm{~S}$ \\
Reconocimiento del éxito personal & 5 & $\mathrm{BS}$ \\
\hline Fuente: Elaboración propia (2009) & &
\end{tabular}

\section{Discusión de resultados}

En relación con la elaboración y validación del instrumento de medición se puede mencionar que alcanzó un alto nivel de validez y confiabilidad, con un Alfa de Cronbach de 0,94 lo que nos indica una alta homogeneidad y equivalencia de respuesta a todos los ítems y para todos los encuestados, por lo tanto este instrumento de medición de la calidad de los servicios estudiantiles puede utilizarse en cualquier institución de educación superior con las adaptaciones pertinentes.

Como consecuencia de lo anterior el Departamento de Ingeniería Industrial dispone entonces de un instrumento válido para evaluar el nivel de satisfacción de los estudiantes, por lo que ofrece la oportunidad de ser aplicado de manera periódica con el fin de mantener una estadística de cómo van evolucionando los índices de valoración así como advertir eventuales cambios en las dimensiones de interés de los estudiantes.

Con respecto al análisis de resultados, se destaca que el año de ingreso de los estudiantes es un componente importante al momento de hablar de satisfacción estudiantil, esto porque a mayor cantidad de años de estudio en el Departamento, mayor es el nivel de satisfacción de los estudiantes. Esta situación puede deberse a múltiples factores, dentro de los cuales el grado de pertenencia con la institución puede alterar favorablemente los resultados, sin embargo puede deberse también a un grado de conocimiento mayor de la Universidad por la experiencia adquirida.

En cuánto a la edad, no es una variable determinante al momento de hablar de satisfacción estudiantil, en este estudio una posible explicación puede ser que las edades eran relativamente homogéneas entre los encuestados no siendo posible discriminar percepciones entre los estudiantes que pertenecen a tramos de edades diferentes. En este sentido, se recomienda para futuras investigaciones considerar dentro de los criterios de selección de la muestra el factor edad, para poder comparar diferentes grupos y poder advertir si efectivamente el factor edad es determinante para tomar decisiones.

La variable género muestra una diferencia en relación a los niveles de satisfacción entre mujeres y hombres. El grupo de mujeres (41.6\% del total entre hombres y mujeres) se muestran más satisfechos estudiantilmente que el grupo de los hombres. Si bien existe esta percepción, al 
profundizar en el análisis se observa que no es una diferencia tan marcada dado que la diferencia está entre una evaluación de "satisfecho" y "bastante satisfecho".

Por otra parte, al analizar los ítems con menor puntuación se observa que un 54.5\% de ellos corresponden a la dimensión "Condiciones básicas de infraestructura", esta situación puede ser reflejo de que en la actualidad el Departamento de Ingeniería Industrial se encuentra en proceso de remodelación, lo cual ha afectado significativamente la calidad de vida de los estudiantes al interior de las dependencias actuales. Se espera que una vez terminadas las obras de re construcción del departamento académico la valoración por parte de los estudiantes aumente significativamente.

Los ítems con mayor valoración corresponden en un 50\% a la dimensión "Trato afectuoso" esto se debe a que la Universidad del Bío Bío y particularmente el Departamento de Ingeniería Industrial, cuenta con funcionarios que brindan un servicio eficiente y particularmente atento, en este caso se destacó el trato afectuoso del personal de biblioteca, del director del departamento, de las secretarias y auxiliares y del personal de apoyo del laboratorio de computación. Lugo le sigue con un menor porcentaje, pero no menos significativos, los ítems relacionados con el acceso a Internet y Equipos computacionales dentro del Departamento de Ingeniería Industrial, el servicio bibliotecario y la incorporación de tecnologías a la sala de clases.

Al analizar los índices de satisfacción total, se observa que sólo un 9\% de los estudiantes encuestados se manifiestan "Poco satisfecho" lo que nos indica que en el Departamento de Ingeniería Civil Industrial los estudiantes encuentran todas aquellas condiciones de "Requisitos Básicos" y "Requisitos de desempeño" se encuentran atendidas satisfactoriamente. Sin embargo, los resultados indican que existe una gran carencia en "Requisitos de Deleite" que son aquellos que generan que el estudiante se sienta gratamente sorprendido con la calidad de servicio y de educación que están recibiendo, por lo tanto aquí es donde se debería generar un gran esfuerzo por parte de los Directivos del Departamento con el fin de mejorar las condiciones de educación de los estudiantes y con esto mejorar el prestigio del departamento y carrera bajo estudio.

Los resultados obtenidos a través del análisis de factores permitieron identificar seis dimensiones de calidad que presentan un alto grado de sensibilidad desde el punto de vista de la satisfacción por parte de los estudiantes de la carrera bajo estudio, estas dimensiones presentan una gran similitud en comparación con las dimensiones teóricas propuestas al inicio de la investigación, lo que nos indica que muy posiblemente fueron debidamente planteados. Además, se puede resaltar el hecho que los atributos referidos a los servicios que presta tanto la institución como el departamento no son atributos que presenten una alta valoración por parte de la población bajo estudio.

Al realizar un análisis comparativo de los resultados arrojados entre este estudio y otro de similares características, también aplicado a una comunidad de estudiantes de Ingeniería Industrial 
(Martínez y Mejías, 2007) se puede deducir que, por una parte que la cantidad de ítemes de preguntas que conformaron las dimensiones de interés en ambos estudios fue de 21 ítemes significativos, sin embargo, el grado de similitud conceptual de esos ítemes de preguntas y sus dimensiones resultantes se estima solo fue del orden del $40 \%$ de similitud entre ambas carreras universitarias. Las principales similitudes entre ambos estudios se encuentran radicadas en temas relacionados con las metodologías de enseñanza, organización académica y empatía entre estudiantes y personal administrativo y académico.

\section{Conclusiones}

De los resultados mostrados, de su análisis y de su discusión, se pueden obtener las siguientes conclusiones sobre la apliación del análsis de factores a un segmento de estudiantes de la carrera de ingeniería civil industrial, de la UBB.

- Se dispone de un instrumento de medición de la satisfacción estudiantil apropiado para la carrera bajo estudio dado que el instrumento alcanzó un alto grado de validez y confiabilidad, con un Alpha de Cronbach tal que puede ser utilizado incluso en otra institución de educación superior de similares características.

- El análisis de factores permitió identificar de manera objetiva seis dimensiones de calidad de interés (Gestión Metodológica, Desarrollo personal, Servicios tecnológicos, Seguridad emocional, Seguridad física y Reconocimiento del éxito personal ) que permitirá enfocar de manera más eficaz futuras acciones en el ámbito de la difusión y marketing asociados a la carrera bajo estudio.

- La técnica de análisis de factores no es difícil de aplicar si se tienen los conocimientos estadísticos mínimos necesarios y existe interés por revisar y analizar diversos estudios disponibles en este ámbito, incluso esta técnica se puede aplicar al interior de departamentos o áreas en empresas de manufactura para evaluar percepciones de grupos de clientes internos.

- La variable “Año de ingreso" fue la única variable que presentó una correlación directa con el nivel de satisfacción estudiantil, ya que se observó que a medida que el estudiante avanza en el desarrollo de su carrera su nivel de satisfacción es mayor.

- El nivel de satisfacción total valorado por los estudiantes, que resultó ser el nivel de "Satisfecho", indica que existe fundamento técnico suficiente que soporta este resultado de valoración de satisfacción y, por otra parte, este nivel de satisfacción puede ser considerado como un dato base para monitorear el nivel de satisfacción en periodos futuros. 


\begin{abstract}
The study aimed to evaluate the satisfaction of a segment of students at higher levels of the industrial engineering degree from the Bio-Bio University, Chile, and identify the dimensions of quality student interest to focus more effectively process support decision making and consequently the process of accreditation of that program. The methodology was the design and implementation of a duly validated survey and application of factor analysis technique. The main results allowed objectively know the current satisfaction level of students under study as well as those more sensitive quality dimensions. The application of the measuring instrument reached a high level of reliability and validity, with a Cronbach Alpha of 0.94 so the instrument can be used at any institution of higher education that offer similar industrial engineering degree.The results indicate an intermediate level of satisfaction within the measurement scale used and the six dimensions relevant to focus the decision making process for the academic program were: Methodological Management, Personal Development, Technological Services, Emotional Security, Security Recognition of physical and personal success.
\end{abstract}

Key-words: factor analysis, quality student dimensions, industrial engineering, student satisfaction.

\title{
Referencias
}

ACOSTA, M., MEJIAS, A. Evaluación de la calidad de servicio en la gestión de suministro de una empresa manufacturera. I SIMPOSIO DE INGENIERÍA INDUSTRIAL, 27-30 de noviembre de 2007. Universidad de Carabobo. Valencia, Venezuela, Anales. Valencia: 1SIII,2007.

BARRIOS, M., MEJIAS, A.. Dimensiones de la calidad de servicio en un centro medico asistencial universitario. . I SIMPOSIO DE INGENIERÍA INDUSTRIAL, 27-30 de noviembre de 2007. Universidad de Carabobo. Valencia, Venezuela, Anales. Valencia: 1SIII, 2007.

BLANCO, A., RIVERO, V., MANEIRO, N. 2009. Calidad de servicio percibida por los clientes residenciales en las oficinas de atención al cliente de una empresa telefónica. III SIMPOSIO DE INGENIERÍA INDUSTRIAL, 2007. Universidad de Boyacá. Colombia, Anales: Boyacá 3SIII, 2009

GARZA, R Y PÉREZ, I. 2007. Aplicación del AHP en la determinación de los requisitos de la calidad. I SIMPOSIO DE INGENIERÍA INDUSTRIAL, 27-30 de noviembre de 2007. Universidad de Carabobo. Valencia, Venezuela, Anales. Valencia: 1SIII, 2007.

ELLIOT, K Y SHIN, D Student satisfaction: an alternative approach to assessing this importat concept. Journal of higher policy and management 24. (2) 197-209, 2002.

GUTIÉREZ, P. Calidad total y productividad, Ed. McGraw Hill, p71-78,2005.

HERNANDEZ,R. Metodología de la Investigación. México, Mc Graw Hill. 505p, 2006.

JOHNSON, D Métodos multivariados aplicados al análisis de datos. México, International Thomson Editores, 2000.

MANRIQUE, S y MEJÍAS, A. Medición de la satisfacción de clientes bancarios universitarios: Una aproximación mediante el análisis de factores. III SIMPOSIO DE INGENIERÍA INDUSTRIAL, 2007. Universidad de Boyacá. Colombia, Anales: Boyacá 3SIII, 2009

MARTINES, D., MEJIAS, A.. Medición de la satisfacción estudiantil en instituciones de educación superior. I SIMPOSIO DE INGENIERÍA INDUSTRIAL, 27-30 de noviembre de 2007. Universidad de Carabobo. Valencia, Venezuela, Anales. Valencia: 1SIII, 2007.

PALACIOS, G. La evaluación de la satisfacción educativa en un enfoque de calidad institucional. Prentice Hall,2002.

PEREZ JUSTE, R.. La calidad de la educación: Hacia una educación de calidad. Gestión, instrumentos y evaluación. Madrid Nancea, 2000

PRAT R y DOVAL E. Construcción y Análisis estadístico de escalas: Análisis Multivariable para las ciencias sociales. Madrid, Pearson Education, 2003.

SIADO, M., MEJÍAS, A.. Evaluación de la calidad de servicio percibida por los clientes de alto consumo de una empresa de servicios eléctricos. I SIMPOSIO DE INGENIERÍA INDUSTRIAL, 27-30 de noviembre de 2007. 
Universidad de Carabobo. Valencia, Venezuela, Anales. Valencia: 1SIII,2007.

TORRES, C.; VARGAS, G., CONTRERAS, S.; BENAVENTE, J. Evaluación de los factores que influyen en el grado de satisfacción de los apoderados en una institución educacional. Revista Theoría. Vol 13: 51-57, 2004.

\section{DADOS DOS AUTORES}

\section{Carlos Torres Navarro}

Universidad del Bío-Bío

Departamento de Ingeniería Industrial

Profesor Asistente A

Av. Collao 1202, Concepción, Chile.

CEP 4081112

Fono: 56-41- 2731381

e-mail: ctorres@ubiobio.cl

\section{Elizabeth Sepúlveda Cárdenas}

Universidad del Bío-Bío

Departamento de Ingeniería Industrial

Ingeniero Civil Industrial

Av. Collao 1202, Concepción, Chile.

CEP 4081112

Fono: 56-41- 2731381

e-mail: elizepul@gmail.com

Recebido para publicação em: 01/10/2009

Aceito para publicação em: 04/12/2009 